\title{
Morphology of Gamma-Ray Halos around Middle-Aged Pulsars: Influence of the Pulsar Proper Motion
}

\author{
Yi Zhang, ${ }^{a, b, *}$ Ruo-Yu Liu, ${ }^{a, b}$ S. Z. Chen ${ }^{c, d}$ and Xiang-Yu Wang ${ }^{a, b}$ \\ ${ }^{a}$ Nanjing University, School of Astronomy and Space Science, \\ Xianlin Road 163, Nanjing, China; \\ ${ }^{b}$ Key laboratory of Modern Astronomy and Astrophysics (Nanjing University),,Ministry of Education, \\ 210023 Nanjing, China \\ ${ }^{c}$ Key Laboratory of Particle Astrophysics, Institute of High Energy Physics, Chinese Academy of Sciences, \\ 100049 Beijing, China \\ ${ }^{d}$ TIANFU Cosmic Ray Research Center, \\ Chengdu, Sichuan, China \\ E-mail: yi_zhang@smail.nju.edu.cn, ryliu@nju.edu.cn, chensz@ihep.ac.cn, \\ xywang@nju.edu.cn
}

Recently, $\gamma$-ray halos of a few degree extension have been detected around two middle-aged pulsars, namely, Geminga and PSR B0656+14, by the High Altitude Water Cherenkov observatory (HAWC). The $\gamma$-ray radiation arise from relativistic electrons that escape the pulsar wind nebula and diffuse in the surrounding medium. The diffusion coefficient is found to be significantly lower than the average value in the Galactic disk. If so, given a typical transverse velocity of $300-500 \mathrm{~km} / \mathrm{s}$ for a pulsar, the displacement of the pulsars due to the proper motion could be important in shaping the morphology of the pulsar halos. Motivated by this, we study the morphology of pulsar halos considering the proper motion of pulsar. We define three evolutionary phases of pulsar halo to categorize its morphological features. We give the maximum separation angle that can be induced by pulsar proper motion and discuss whether the extended sources observed by HAWC and LHAASO can be associated with pulsar. Generally we do not expect to observe the separation between distant pulsar and halo above $10 \mathrm{TeV}$ with LHAASO or HAWC. Our result can help to interpret the origins of the observed extended sources at very high energies.

$37^{\text {th }}$ International Cosmic Ray Conference (ICRC 2021)

July 12th - 23rd, 2021

Online - Berlin, Germany

\footnotetext{
${ }^{*}$ Presenter
} 


\section{Introduction}

Pulsar wind nebulae $(\mathrm{PWNe})$ are bubbles of relativistic electrons and positrons accelerated when a pulsar's relativistic wind interacts with its environment[e.g. 1]. Since a pulsar is formed in a SN explosion, the pulsar and its PWN are initially surrounded by an expanding SNR. The expanding supernova shell is decelerating as it sweeps up the surrounding ISM. Due to the asymmetry in the SN explosion, the pulsar has a natal velocity and ultimately penetrates and escapes the shell. Then the pulsar moves through the ambient ISM with proper velocity $v_{\mathrm{p}}$ while the PWN at this stage is compact and filled with recently injected particles.

The High-Altitude Water Cherenkov Observatory (HAWC) recently reported discovery of spatially extended $\mathrm{TeV}$ sources surrounding two middle-aged $\left(t_{\text {age }}=100-400 \mathrm{kyr}\right)$ pulsars, namely, Geminga and PSR B0656+14 [2]. The physical extension of the sources is about $30 \mathrm{pc}$, which is much larger than the size of the PWN of the corresponding pulsars. The intensity profile of the $\mathrm{TeV}$ sources can be explained with the inverse Compton (IC) scatterings of diffusing electrons that escaped from the PWN on the cosmic microwave background (CMB) and the interstellar radiation field (ISRF) [2-8]. In addition to Geminga and PSR B0656+14, many more such pulsar halos could have been already detected by instruments such as HAWC, MILAGRO, HESS, Fermi-LAT and LHAASO [9-11].

The morphology of the $\gamma$-ray halos is an important property for identifying pulsar halos. A pulsar's proper motion may lead to a displacement of about $80\left(v_{\mathrm{p}} / 400 \mathrm{~km} \mathrm{~s}^{-1}\right)\left(t_{\text {age }} / 200 \mathrm{kyr}\right) \mathrm{pc}$ from its birth place. Such a displacement could be larger than the spatial extension of the $\mathrm{TeV}$ halo for middle-aged pulsars, so it may largely affect the $\gamma$-ray morphology. For example, some research groups have discussed about the influence of Geminga proper motion to the morphology of its $\gamma$-ray halo $[4,7,8]$. Various factors such as continuous injection of electrons, diffusion and cooling of electrons, magnetic and radiation field and the limit of the angular resolution of instruments (i.e., the PSF) could influence the expected offset and complicate the identification of pulsar halos, while a detailed evaluation on the influence of these factors has not been performed.

Motivated by this, we study the effect of pulsar's proper motion on the morphology of pulsar halos at $\mathrm{GeV}$ and $\mathrm{TeV}$ band. We focus on pulsar with an age of $t_{\mathrm{age}} \geq 100 \mathrm{kyr}$, when the electrons that escape the PWN are diffusing in the ISM. The rest of the paper is organized as follows. In Section 2, we introduce our model for the $\gamma$-ray emission of the pulsar halo. In Section 3, we define three evolutionary phases of pulsar halo. In section 4 , we give the maximum separation angle that can be induced by pulsar proper motion. In section 5, we discuss whether the observed sources by HAWC and LHAASO can be associated with pulsar.

\section{Model for the $\gamma$-ray emission of a pulsar halo}

In the model of pulsar halo considered in this work, relativistic electrons are continuously injected into the ambient ISM of a pulsar. We assume that the evolution of the total electron injection rate follows the pulsar's spin-down history as

$$
L_{e}(t)=\eta_{e} L_{i}\left(1+t / \tau_{0}\right)^{-(n+1) /(n-1)},
$$

where $t$ is the time after the pulsar's birth. For the benchmark pulsar, we refer to the parameters of Geminga and take $n=3 . \eta_{e}=1$ is the fraction of the spin-down energy converted into relativistic 
electrons, $L_{i}=1.7 \times 10^{37} \mathrm{erg} / \mathrm{s}$ is the initial spin-down luminosity of the pulsar, $\tau_{0}=15 \mathrm{kyr}$ is the initial spin-down timescale with $\tau_{c}$ being the "characteristic age". About the age of the benchmark pulsar, we consider two cases, one is $t_{\text {age }}=327 \mathrm{kyr}$, corresponding to the age of Geminga, and the other is $t_{\text {age }}=100 \mathrm{kyr}$, for which the most energetic electrons have not cooled to several TeV.

We assume the injection electron spectrum to be a power-law function with an exponential cutoff, i.e., $Q_{e}\left(E_{e}, t\right)=Q_{0}(t) E_{e}^{-\gamma_{e}} \exp \left(-\frac{E_{e}}{E_{c}}\right)$, where $Q_{0}(t)$ is the normalization factor which can be determined by $\int E_{e} Q_{e}\left(E_{e}, t\right) d E_{e}=L_{e}(t)$. We consider $\gamma_{e}=2$ and $E_{c}=400 \mathrm{TeV}$ as reference parameters.

The transport equation of electrons injected from a point source located at $\boldsymbol{r}_{s}$ is

$$
\frac{\partial n_{e}}{\partial t}=D\left(E_{e}\right) \nabla_{\boldsymbol{r}}^{2} n_{e}+\frac{\partial\left[b\left(\boldsymbol{r}, E_{e}, t\right) n_{e}\right]}{\partial E_{e}}+Q_{e}\left(E_{e}, t\right) \delta^{3}\left(\boldsymbol{r}-\boldsymbol{r}_{s}\right),
$$

where $n_{e}\left(\boldsymbol{r}, E_{e}, t\right)$ is the differential electron density at time $t$ and position $\boldsymbol{r}$, and $D\left(E_{e}\right)$ is the diffusion coefficient assuming isotropic diffusion, which is assumed to be spatially homogeneous and scales with the energy as $D\left(E_{e}\right)=D_{0}\left(E_{e} / 1 \mathrm{GeV}\right)^{1 / 3} . b\left(\boldsymbol{r}, E_{e}, t\right)$ is the energy loss rate of electrons during the propagation due to the synchrotron and IC radiation. We here consider a homogeneous and constant magnetic field and radiation field. We assume a homogeneous magnetic field of $B=3 \mu \mathrm{G}$ and consider four blackbody/greybody components around the pulsar as: $\mathrm{CMB}(T=2.73 \mathrm{~K}$ and $\left.U=0.25 \mathrm{eVcm}^{-3}\right)$, far-infrared radiation (FIR) field $\left(T=40 \mathrm{~K}\right.$ and $\left.U=1 \mathrm{eVcm}^{-3}\right)$, near-infrared radiation field $\left(T=500 \mathrm{~K}\right.$ and $\left.U=0.4 \mathrm{eVcm}^{-3}\right)$ and visible light radiation field $(T=3500 \mathrm{~K}$ and $\left.U=1.9 \mathrm{eVcm}^{-3}\right)$.

The present-day $\left(t=t_{\text {age }}\right)$ density of electrons with energy $E_{e}$ at a radius $r$ away from the pulsar can be calculated by

$$
n_{e}\left(\boldsymbol{r}, E_{e}\right)=\int_{0}^{t_{\mathrm{age}}} d t^{\prime} Q_{e}\left(\mathcal{E}_{e}^{\prime}\left(t^{\prime}\right), t^{\prime}\right) \times \frac{\exp \left[-\left(\boldsymbol{r}-\boldsymbol{r}_{s}\left(t^{\prime}\right)\right)^{2} / 4 \lambda\left(E_{e}, t^{\prime}\right)\right]}{\left(4 \pi \lambda\left(E_{e}, t^{\prime}\right)\right)^{3 / 2}} \frac{d \mathcal{E}_{e}^{\prime}\left(t^{\prime}\right)}{d E_{e}},
$$

where $\lambda\left(E_{e}, t^{\prime}\right)=\int_{t^{\prime}}^{t_{a g e}} D\left(E_{e}\left(t^{\prime \prime}\right)\right) d t^{\prime \prime}$ and $\mathcal{E}_{e}^{\prime}$ is the electron energy at injection. We take the cylindrical coordinate system and set the direction of the proper motion as the $x$-axis. The present pulsar position is set to $(0,0,0)$ and then the historical position of the pulsar can be give by $\boldsymbol{r}_{s}=\left(v_{\mathrm{p}}\left(t_{\text {age }}-t\right), 0,0\right)$. We take the angle between pulsar proper motion direction and LOS as $\psi=90^{\circ}$ and take $v_{\mathrm{p}}=400 \mathrm{~km} / \mathrm{s}$, namely the transverse velocity of pulsar $v_{\text {tr }}$, to exhibit the morphology of pulsar halo at different energies.

We then calculate the $\gamma$-ray emission produced by the IC process of electrons and integrate them over the LOS at different viewing angle [6]. We obtain the $\gamma$-ray intensity at a polar angle $\theta$ from the pulsar and an azimuth angle $\phi$ ( $\phi=0$ points to the opposite direction of the proper motion) $I_{\gamma}^{\prime}\left(E_{\gamma}, \theta, \phi\right)$, which describes the intrinsic morphology of the $\gamma$-ray halo.

We convolve $I_{\gamma}^{\prime}$ with the PSF of various instruments at different energies to simulate the observed intensity map or the morphology of the $\gamma$-ray halo by the instruments. The $\gamma$-ray intensity map after the convolution with the PSF is then given by

$$
I_{\gamma}\left(E_{\gamma}, \theta, \phi\right)=\iint \frac{1}{2 \pi \sigma^{2}} \exp \left(-\frac{l^{\prime 2}}{2 \sigma^{2}}\right) I_{\gamma}^{\prime}\left(E_{\gamma}, \theta^{\prime}, \phi^{\prime}\right) \sin \theta^{\prime} d \theta^{\prime} d \phi^{\prime} .
$$

where $l^{\prime}=\cos \theta \cos \theta^{\prime}+\sin \theta \sin \theta^{\prime} \cos \left(\phi-\phi^{\prime}\right)$ is the angular distance between the point $(\theta, \phi)$ and the point $\left(\theta^{\prime}, \phi^{\prime}\right)$ in the celestial plane, and $\sigma\left(E_{\gamma}\right)$ is the size of the PSF as a function of $\gamma$-ray energy. 


\section{Evolutionary phases of pulsar halo}

To understand the energy-dependent morphology of the pulsar halo, we define two critical timescales. One is $t_{\mathrm{pd}}=80\left(E_{e} / 1 \mathrm{TeV}\right)^{1 / 3}\left(D_{0} / 10^{26} \mathrm{~cm}^{2} \mathrm{~s}^{-1}\right)\left(v_{\mathrm{tr}} / 400 \mathrm{~km} / \mathrm{s}\right)^{-2} \mathrm{kyr}$, the time when the electron's diffusion distance $(2 \sqrt{D t})$ is equal to the distance that the pulsar has moved $\left(v_{\mathrm{tr}} t\right)$. The other one $t_{\mathrm{c}}$ is the time in which an electron cools from energy $E_{c}$ (the cutoff energy in the injection spectrum) to certain energy $E_{e}$. Then we divide the morphological evolution of the pulsar halo into three phases, based on these two timescales. This definition of phases can help to generalize different feature and origin of different pulsar halo morphology. This simple physical definition works well for pulsars with $n>2.5$ and $\tau_{0}>5 \mathrm{kyr}$. The three phases of pulsar halo evolution can be given by:

PHASE I: $t_{\text {age }}<t_{\mathrm{pd}}, t_{\mathrm{age}}<t_{\mathrm{c}}$. The electrons injected at early epoch with a high luminosity haven't cooled and pulsar's displacement due to proper motion is still within the diffusion length of these relic electrons. The relic electrons produce bright radiation around the original position of the pulsar. Since relic electrons have diffused to a larger distance than the pulsar has travelled, the emissions of electrons injected at different epoch overlap with each other and yield a single and broad peak in the intensity map.

PHASE II: $t_{\mathrm{pd}}<t_{\mathrm{age}}<t_{\mathrm{c}}$. Since the displacement of the pulsar from its original position is proportional to $t$ while electron's diffusion distance is proportional to $t^{1 / 2}$, the pulsar eventually goes beyond the diffusion length of electrons injected at early epoch, and the fresh electrons yield elongated bright regions along the pulsar trajectory. The pulsar halo is highly asymmetrical, doublepeak or single-peak with an extension towards the direction $x>0$. The relative brightness at the original position and the current position of the pulsar depends on $L_{e}(t)$.

PHASE III: $t_{\mathrm{age}}>t_{\mathrm{c}}$. Electrons injected at early epoch lost most energy and only recently injected electrons can produce $\gamma$-ray emission. The morphology of pulsar halo is again single-peak and compact, with a rough circular symmetry with respect to the current position of the pulsar.

Note that in the transition stage from one phase to another phase (i.e., $t_{\mathrm{age}} \sim t_{\mathrm{pd}}, t_{\mathrm{pd}} \sim t_{\mathrm{c}}$ ), the feature of a certain phase mentioned above is not distinct and relies on the relative contribution from relic and newly injected electrons, i.e. $L_{e}(t)$. We show in Fig.1 the intensity profiles of the pulsar halo of the benchmark pulsar $\left(t_{\text {age }}=100 \mathrm{kyr}\right)$ and assume $v_{\text {tr }}=200 \mathrm{~km} / \mathrm{s}$ and $800 \mathrm{~km} / \mathrm{s}$ respectively, to compare the feature of pulsar halo at PHASE I with that at PHASE II. At this age, the pulsar halo at $10 \mathrm{GeV}$ and $1 \mathrm{TeV}$ with $v_{\text {tr }}=200 \mathrm{~km} / \mathrm{s}$ belongs to PHASE I (dashed curves) while those with $v_{\text {tr }}=800 \mathrm{~km} / \mathrm{s}$ belong to PHASE II (curve lines). As we discussed above, the pulsar halo at PHASE I is single-peak. The pulsar halo at PHASE II is more asymmetrical with an extension towards the direction of the pulsar's initial position.

\section{Maximum separation angle}

We now look into the expected offset of the pulsar and the $\gamma$-ray halo induced by pulsar proper motion. We define two kinds of offsets: one is the separation between the pulsar' current position and the centroid of the halo (denoted by $\Theta$ ), and the other is the separation between the pulsar and the position of the brightest point or the intensity peak of the halo (denoted by $\left.\Theta^{\prime}\right)$. The latter is straightforward to obtain. To get the former, we use a 2D Gaussian template 


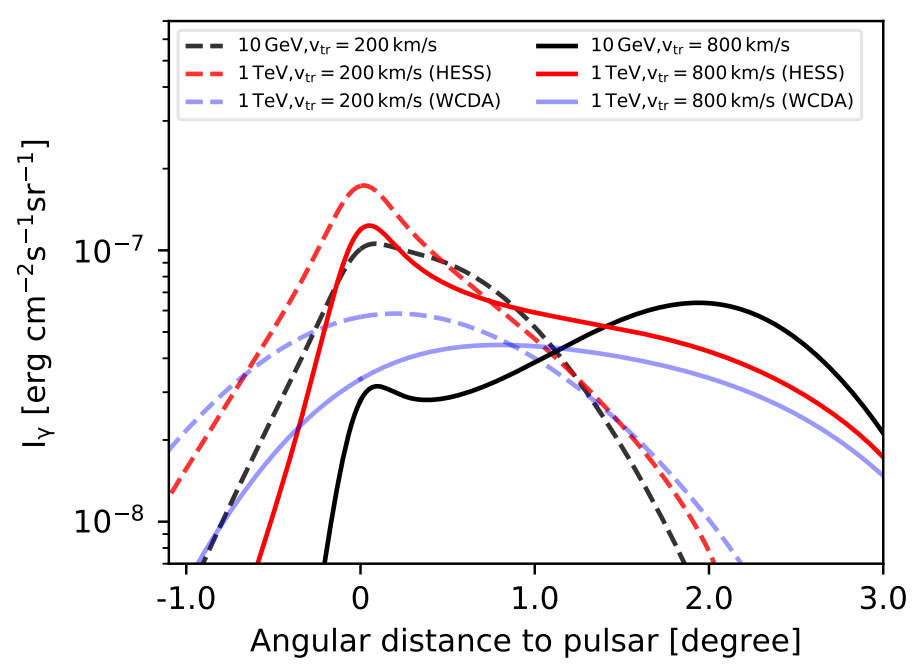

Figure 1: The $\gamma$-ray intensity profile of benchmark pulsar $\left(t_{\mathrm{age}}=100 \mathrm{kyr}\right)$, but with $v_{\mathrm{tr}}=200 \mathrm{~km} / \mathrm{s}$ and $800 \mathrm{~km} / \mathrm{s}$, after convolving with the PSF of Fermi-LAT $(10 \mathrm{GeV})$, HESS (1 TeV) and LHAASO(WCDA) $(1 \mathrm{TeV})$.

as $I_{G}(\boldsymbol{r})=\left(N_{0} / 2 \pi \sigma_{0}^{2}\right) \exp \left[-\left(\boldsymbol{r}-\boldsymbol{r}_{c}\right)^{2} / 2 \sigma_{0}^{2}\right]$, where $\boldsymbol{r}_{c}$ is its center, to model the PSF-convolved intensity map. We set $N_{0}, \sigma_{0}$ and $\boldsymbol{r}_{c}$ as free parameters, and search for the centroid of the halo $\left(\boldsymbol{r}_{c}\right)$ by minimizing the chi-square $\chi^{2}=\sum_{\boldsymbol{r}}\left(I_{G}-I_{\gamma}\right)^{2} / I_{\gamma}$.

As the pulsar wind luminosity decreases with time, the maximum energy of electrons achievable in the termination shock may also decreases with time. When the maximum energy is below certain energy, we can regard the injection of electrons of this energy being ceased. The termination of injection would cause a larger offset because electrons are not injected at the position close to the present position of the pulsar. However, if the injection stops too early, i.e., the time experienced from the epoch of the injection termination to the present time, denoted by $t_{\mathrm{s}}$, is longer than the cooling timescale $t_{\mathrm{c}}$, we do not expect to see the emission of electrons at present. Therefore, we expect the most favorable condition for a large offset to be $t_{\mathrm{s}} \sim t_{\mathrm{c}}$. We then calculate the evolution of the pulsar halo at each energy with turning off the electron injection at $t=t_{\mathrm{age}}-t_{\mathrm{c}}$ to maximize the separation angle. The result is shown in Fig. 2, assuming $B=3 \mu \mathrm{G}$. The offset obtained in this condition $\left(\Theta_{\max }\right)$ is independent on the electron injection history. We find that the $\Theta_{\max }-E_{\gamma}$ relation in the range of $1-100 \mathrm{TeV}$ can be empirically depicted by

$$
\Theta_{\max }=3^{\circ}\left(\frac{E_{\gamma}}{1 \mathrm{TeV}}\right)^{-0.77}\left(\frac{v_{\mathrm{tr}}}{400 \mathrm{~km} / \mathrm{s}}\right)\left(\frac{d}{2 \mathrm{kpc}}\right)^{-1},
$$

We note that the offset above $100 \mathrm{TeV}$ is quite small due to the rapid cooling of ultra-high energy electrons and is difficult to be resolved by the current $\gamma$-ray instrument, i.e., LHAASO-KM2A, unless the pulsar is located sufficiently close to Earth (e.g., at a distance of $\sim 100 \mathrm{pc}$ from Earth) and has a high proper velocity (e.g., $\gtrsim 1000 \mathrm{~km} / \mathrm{s}$ ). 


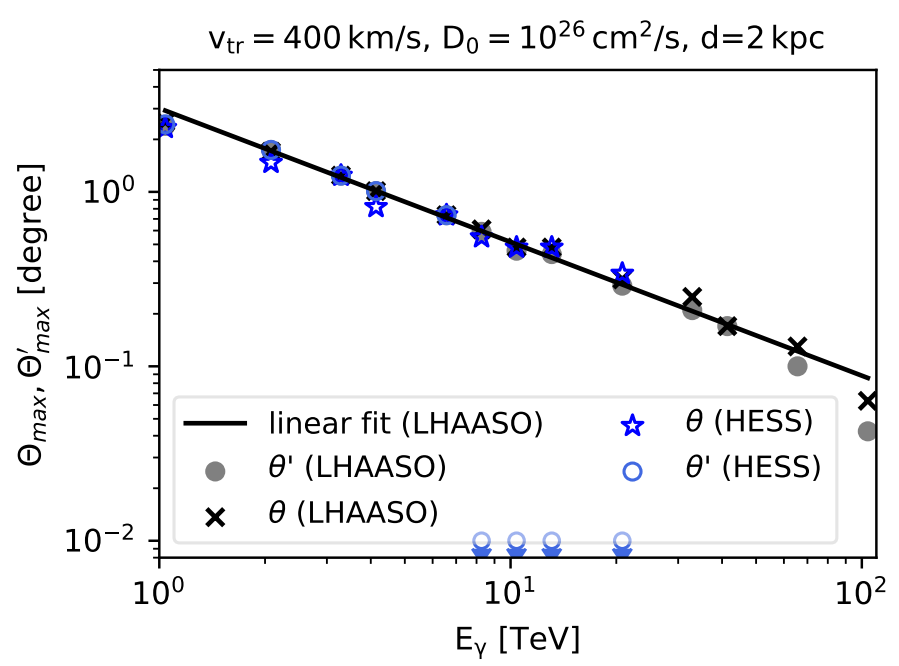

Figure 2: The maximum separation angle $\Theta_{\max }$ and $\Theta_{\max }^{\prime}$ at different energies that can be produced by a pulsar located at $2 \mathrm{kpc}$ with a proper velocity of $v_{\mathrm{tr}}=400 \mathrm{~km} / \mathrm{s}$.

Table 1: $3 \mathrm{HWC}$ and LHAASO sources with TeV halo candidate pulsars

\begin{tabular}{ccccccc}
\hline \hline 3HWC & Pulsar & $\tau_{c}(\mathrm{kyr})$ & $\mathrm{d}(\mathrm{kpc})$ & $v_{\text {tr }}(\mathrm{km} / \mathrm{s})$ & $\theta_{\text {obs }}\left({ }^{\circ}\right)$ & Comment \\
\hline $\mathrm{J} 0540+228$ & B0540+23 & 253 & 1.56 & 215 & 0.83 & $\mathrm{~B}<1 \mu \mathrm{G}$ or $n<2$ \\
$\mathrm{~J} 0543+231$ & B0540+23 & 253 & 1.56 & 215 & 0.36 & Unaligned \\
$\mathrm{J} 0631+169$ & $\mathrm{~J} 0633+1746$ & 342 & 0.19 & 128 & 0.95 & Possible \\
$\mathrm{J} 0634+180$ & $\mathrm{~J} 0633+1746$ & 342 & 0.19 & 128 & 0.38 & Unaligned \\
$\mathrm{J} 0659+147$ & B0656+14 & 111 & 0.29 & 60 & 0.51 & Unaligned \\
$\mathrm{J} 0702+147$ & B0656+14 & 111 & 0.29 & 60 & 0.77 & Unaligned \\
\hline \hline LHAASO & Pulsar & $\tau_{c}(\mathrm{kyr})$ & $\mathrm{d}(\mathrm{kpc})$ & $v_{\text {tr }}(\mathrm{km} / \mathrm{s})$ & $\theta_{\text {obs }}\left({ }^{\circ}\right)$ & Comment \\
\hline J2032+4102 & J2032+4127 & 201 & 1.4 & 20.4 & 0.42 & Impossible \\
$\mathrm{J} 1929+1745$ & $\mathrm{~J} 1928+1746$ & 82.6 & 4.6 & - & 0.25 & $v_{\text {tr }}>2700 \mathrm{~km} / \mathrm{s}$ \\
\hline
\end{tabular}

Pulsar halo candidates in 3HWC and LHAASO source list (column 1), related pulsars (column 2), the pulsar's characteristic age (column 3), distance (column 4), velocity (column 5) and observed separation between pulsar and source (column 6). The properties of pulsars are from the ATNF pulsar catalog [12] or otherwise specified. Our brief comment on whether the separation between the pulsar and the source can be explained by pulsar proper motion is given in the last column.

\section{Application to the observation}

As we discussed above, it is increasingly difficult to produce resolvable offset between the center of the pulsar halo and the pulsar by the proper motion at higher energy. However, offsets at such a high energy between extended $\mathrm{TeV}$ sources and the positions of associated middle-aged pulsars have already been observed by HAWC and LHAASO.

The 3HWC catalogue gives 12 extended TeV sources and the separation between the sources and their candidate pulsars [9]. We list the 3HWC sources and potential pulsars in Table. 1. If we 
assume the separation is caused by pulsar proper motion, four 3HWC sources can be excluded from the association with candidate pulsars due to the not aligned pulsar motion direction. To associate 3HWC J0540+228 with pulsar B0540+23, the magnetic field of the ISM needs to be smaller than $1 \mu \mathrm{G}$, or the braking index of pulsar is at least smaller than 2 . For other eight $3 \mathrm{HWC}$ sources, the association is possible with proper combination of parameters and we need further knowledge of the proper motion velocity of their candidate pulsars to give a robust judgement.

Very recently, LHAASO reported discovery of $12 \gamma$-ray sources above $100 \mathrm{TeV}$ with more than $7 \sigma$ statistical significance [11]. Among them, two pulsar halo candidates, i.e. LHAASO J2032+4102 and LHAASO J1929+1745, are possibly associated with middle-aged pulsar PSR J2032+4127 and PSR J1928+1746 respectively. We can compare the observed separation with the theoretical maximum $\Theta$ (Eq.5) to quickly estimate whether the association could be true. We find that LHAASO J2032+4102 is impossible to be entirely associated with PSR J2032+4127 due to the small $v_{\text {tr }}$ of pulsar, and the association of LHAASO J1929+1745 with PSR J1928+1746 requires an extremely large $v_{\text {tr. }}$ The results are also shown in Table. 1.

\section{Conclusions}

In this paper, we studied the theoretical $\gamma$-ray morphology of a pulsar halo and the spatial offset between the halo and its associated pulsar considering the pulsar's proper motion. We divide the evolution of the pulsar halo into three characteristic phases, that can help to interpret the origin of the pulsar halo. We defined two kinds of offsets between the pulsar halo and the pulsar. We found that the maximum separation angle can be given by $\Theta_{\max }=3^{\circ}\left(E_{\gamma} / 1 \mathrm{TeV}\right)^{-0.77}\left(v_{\mathrm{tr}} / 400 \mathrm{~km} \mathrm{~s}^{-1}\right)(d / 2 \mathrm{kpc})^{-1}$ empirically, assuming $B=3 \mu \mathrm{G}$. Generally, above $10 \mathrm{TeV}$, the offset between extended sources observed by HAWC and LHAASO and candidate pulsar are hard to be explained by pulsar proper motion. If a $3 \mathrm{HWC}$ source or a LHAASO source is truly a pulsar halo but the spatial offset between the source and the related pulsar is very large, we have to resort to more complicated but probably realistic scenarios, such as the anisotropic particle diffusion scenario if the geometric configuration of the magnetic field in the surrounding ISM of the pulsars is not chaotic [6]. Of course, the source is not necessarily a pulsar halo. Some sources could have an asymmetric morphology intrinsically. With certain specific conditions, a 10 kyr-aged PWN could vigorously expand [13] while the asymmetric reverse shock arising from the supernova ejecta could crush one side of the PWN [14], and/or electrons preferentially escape from one side of the PWN due to the geometry of the magnetic field in the PWN [15]. Alternatively, the source could be composed of multiple origins. There are more than one source candidates around the two LHAASO sources [11]. Multiwavelength observations with high angular resolution will be crucial to reveal the true nature of the sources.

\section{References}

[1] B.M. Gaensler and P.O. Slane, The Evolution and Structure of Pulsar Wind Nebulae, 44 (2006) 17 [astro-ph/0601081].

[2] A.U. Abeysekara, A. Albert, R. Alfaro, C. Alvarez, J.D. Álvarez and et al., Extended gamma-ray sources around pulsars constrain the origin of the positron flux at Earth, Science 358 (2017) 911 [1711.06223]. 
[3] R. López-Coto and G. Giacinti, Constraining the properties of the magnetic turbulence in the Geminga region using HAWC $\gamma$-ray data, 479 (2018) 4526 [1712 . 04373].

[4] X. Tang and T. Piran, Positron flux and $\gamma$-ray emission from Geminga pulsar and pulsar wind nebula, 484 (2019) 3491 [1808.02445].

[5] R.-Y. Liu, C. Ge, X.-N. Sun and X.-Y. Wang, Constraining the Magnetic Field in the TeV Halo of Geminga with X-Ray Observations, 875 (2019) 149 [1904 . 11438].

[6] R.-Y. Liu, H. Yan and H. Zhang, Understanding the Multiwavelength Observation of Geminga's Tev Halo: The Role of Anisotropic Diffusion of Particles, 123 (2019) 221103.

[7] G. Jóhannesson, T.A. Porter and I.V. Moskalenko, Cosmic-Ray Propagation in Light of the Recent Observation of Geminga, 879 (2019) 91 [1903.05509].

[8] M. Di Mauro, S. Manconi and F. Donato, Detection of a $\gamma$-ray halo around Geminga with the Fermi-LAT data and implications for the positron flux, 100 (2019) 123015 [1903.05647].

[9] A. Albert, R. Alfaro, C. Alvarez, J.R.A. Camacho, J.C. Arteaga-Velázquez, K.P. Arunbabu et al., 3HWC: The Third HAWC Catalog of Very-High-Energy Gamma-ray Sources, arXiv e-prints (2020) arXiv:2007.08582 [2007.08582].

[10] H. E. S. S. Collaboration, H. Abdalla, A. Abramowski, F. Aharonian, F. Ait Benkhali, E.O. Angüner et al., The H.E.S.S. Galactic plane survey, 612 (2018) A1 [1804.02432].

[11] LHAASO Collaboration, Ultrahigh-energy photons up to 1.4 petaelectronvolts from 12 $\gamma$-ray Galactic sources, Nature 594 (2021) 33.

[12] R.N. Manchester, G.B. Hobbs, A. Teoh and M. Hobbs, The Australia Telescope National Facility Pulsar Catalogue, 129 (2005) 1993 [astro-ph/0412641].

[13] D. Khangulyan, A.V. Koldoba, G.V. Ustyugova, S.V. Bogovalov and F. Aharonian, On the Anomalously Large Extension of the Pulsar Wind Nebula HESS J1825-137, 860 (2018) 59 [1712.10161].

[14] F. Aharonian, A.G. Akhperjanian, A.R. Bazer-Bachi, M. Beilicke, W. Benbow, D. Berge et al., Energy dependent $\gamma$-ray morphology in the pulsar wind nebula HESS J1825-137, 460 (2006) 365 [astro-ph/0607548].

[15] R.-Y. Liu and H. Yan, On the unusually large spatial extent of the TeV nebula HESS J1825-137: implication from the energy-dependent morphology, 494 (2020) 2618 [1907.02498]. 